\title{
FINITE ELEMENT APPROXIMATION OF A TIME-DEPENDENT TOPOLOGY OPTIMIZATION PROBLEM
}

\author{
M. Bruggi ${ }^{1}$, N. Parolini ${ }^{2}$, F. Regazzoni ${ }^{2}$ and M. Verani ${ }^{2}$ \\ ${ }^{1}$ Dipartimento di Ingegneria Civile e Ambientale, Politecnico di Milano \\ P.zza Leonardo da Vinci 32, 20133 Milano, Italia. \\ e-mail: matteo.bruggi@polimi.it \\ ${ }^{2}$ MOX - Dipartimento di Matematica, Politecnico di Milano \\ P.zza Leonardo da Vinci 32, 20133 Milano, Italia. \\ e-mail: \{nicola.parolini,marco.verani\}@ polimi.it, francesco1.regazzoni@mail.polimi.it
}

Keywords: Topology optimization, finite element method, additive manufacturing

\begin{abstract}
In most additive manufacturing technologies, support structures are required to sustain overhanging surfaces. These additional structures have negative effects on both processing time and material consumption. Moreover, additional post-processing effort is required for their removal. Therefore, reducing the use of the supports would have a beneficial impact on the overall manufacturing process.

An optimization procedure aiming at the optimal placement (and design) of the supports in additive manufacturing should include the intrinsic time-dependent nature of the process. More precisely, it should take into account not only the final configuration, but all the intermediate shapes that are obtained during the additive process. As a consequence, we believe that it is necessary to go beyond standard topology optimization methods, where typically only the final shape is optimized.

The model proposed in this work relies on the solution of a time-dependent minimal compliance problem based on the classical Solid Isotropic Material with Penalization (SIMP) method. In particular, we first introduce a continuous optimization problem with the state equation defined as the time-integral of a linear elasticity problem on a space-time domain. The objective functional is given by the mean compliance over a time interval. The optimality conditions for this optimization problem are then derived and a fixed-point algorithm is introduced for the iterative computation of the optimal solution.

Numerical examples showing the differences between a standard SIMP method, which only optimizes the shape at the final time, and the proposed time-dependent approach are presented and discussed.
\end{abstract}




\section{INTRODUCTION}

Additive manufacturing, also known as 3D printing, is widely used to create prototypes from digital models. Successive layers of material are laid down by a three-dimensional printer under computer control. In most additive manufacturing technologies, support structures are required to sustain overhanging surfaces. Up to now, not any shape or geometry can be printed in real time, because of the need for providing a suitable set of supports when synthesizing the threedimensional object. Support structures remarkably affect not only the processing times but also the material consumption. Therefore, their rational use would greatly improve the overall process of 3D printing.

Powerful numerical tools that go under the name of methods of topology optimization are rapidly developing in many branches of engineering to find optimal layouts that maximize any kind of performance, see [1]. The classical formulation searches for the distribution of the available amount of isotropic material such that the so-called compliance (twice the elastic strain energy computed at equilibrium) is minimized. A suitable interpolation depending on the local values of the unknown density field penalizes the mechanical properties of the elastic body to achieve 0-1 solutions, see e.g. the well-known SIMP (Solid Isotropic Material with Penalization) [2]. Methods of mathematical programming are adopted to solve the arising minimization problem, see [3], generally resorting to the adoption of the finite element method to solve the equilibrium equation and compute the objective function and its sensitivity with respect to the design variables.

Additive manufacturing is a fertile area of research for topology optimization. According to the recent and comprehensive contribution in [4], additive manufacturing fills the gap between topology optimization and application, since any computed optimal design can be printed with minimal limitations on its complexity. Among the others, [5] investigates the issues and opportunities for the application of topology optimization for 3D printing, addressing the production of meso-scale structures to cope with intermediated density regions, whereas [6] copes with the multiple-material topology optimization of compliant mechanisms created via 3D printing.

Topology optimization can be used not only to generate optimal objects to be printed via additive manufacturing, but even to optimize support structures. An optimization procedure aiming at the optimal placement (and design) of the supports in additive manufacturing should include the inherent time-dependent nature of the process. More precisely, it should account not only for the final configuration, but also for all the intermediate shapes that are handled during the printing.

Goal of this work is to propose a new approach to this problem, resorting to the solution of a time-dependent minimal compliance formulation based on the classical SIMP. More precisely, a continuous optimization problem adopting a state equation defined as the time-integral of a linear elasticity problem on a space-time domain is formulated. The objective function is given by the time-averaged compliance, whereas the optimality conditions for this optimization problem are derived and a fixed-point algorithm is introduced for the iterative computation of the optimal solution. The discretization of the optimization problem is finally obtained by considering $n$ intermediate time instants $\left(t_{i}\right)$ (and the corresponding spatial domains $\Omega\left(t_{i}\right)$ ) and solving a sequence of linear elasticity problems on $\Omega\left(t_{i}\right)$ with the finite element method.

The outline of the paper is as follows. Sections 2 and 3 define the topology optimization problem to design the supports of a 3D printed object having mean minimum compliance over a time interval, in the continuous and discrete form respectively. Section 4 provides numerical examples showing the differences between a standard SIMP method, which only optimizes the 


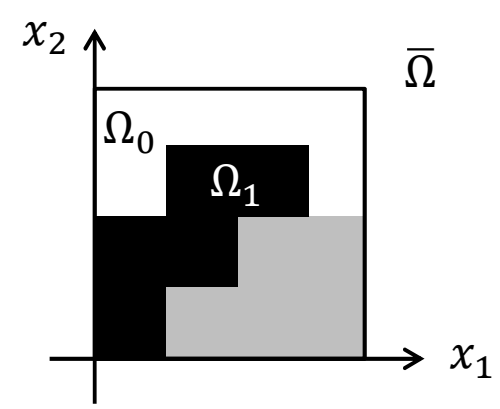

a)

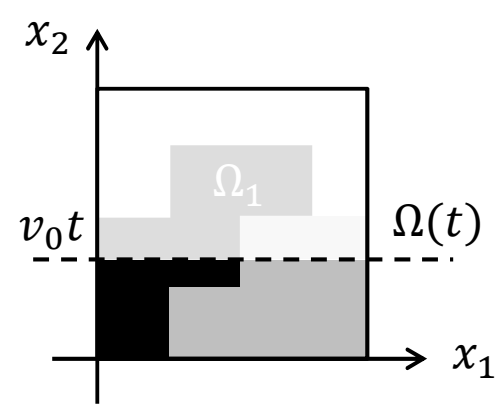

b)

Figure 1: a) Reference space domain. b) Space domain relative to time $t . \Omega(t)$ is located below the dotted line, corresponding to the height $v_{0} t$ ).

shape at the final time, and the proposed time-dependent approach. Section 5 provides final remarks on the presented preliminary simulations and outlines the ongoing research.

\section{THE OPTIMIZATION PROBLEM}

The objective of this work is to define a topology optimization problem to design the supports $\mathcal{S}$ of a 3D printed object $\mathcal{O}$ so that the manufactured item (i.e. $\mathcal{O} \cup \mathcal{S}$ ) exhibits the minimum mean compliance over a given time interval $I=[0, T]$, being $T$ the duration of the production process. To achieve this goal, we will first introduce a suitable time-dependent domain together with a linear elasticity state problem governing the displacement $\mathbf{u}(t)$ of the manufactured item at each time-instant $t$.

Let us consider an hold-all cylindrical space domain $\Omega=E \times(0, h) \subset \mathbb{R}^{d-1} \times \mathbb{R}$, with $d=2,3$ and $E$ a subset of $\mathbb{R}^{d-1}$. Each point in $\Omega$ reads as $\mathbf{x}=\left(\mathbf{x}^{*}, y\right)$, where $\mathbf{x}^{*}$ denotes the planar component while $y$ is the vertical one. Once the printing process is complete, i.e. for $t=T$, the target object $\mathcal{O}$ will occupy a certain subset $\Omega_{1} \subset \Omega$, while for $t<T$ it will occupy intermediate configurations $\Omega_{1}(t)$ such that $\Omega_{1}(t) \subset \Omega$. In view of the above discussion, the value $h$ represents the height of the object at the final time $T$. For future use, we also introduce the subdomain $\Omega_{0} \subset \Omega$ identifying the region where $a$ priori the user does not want to introduce any support. Next, we introduce a time-dependent domain $\Omega(t)$ that changes during the additive manufacturing process and represents the region where the 3D printer can add material (either belonging to the object or to the supports). We assume that $\Omega(t)$ grows in the direction given by the coordinate $y$ with constant velocity $v_{0}$, i.e. $\Omega(t)=\left\{\left(\mathbf{x}^{*}, y\right) \in E \times(0, y): 0<y<v_{0} t\right\}$. Accordingly, we have $\Omega_{1}(t)=\Omega(t) \cap \Omega_{1}$ (see Figure 1 1 . Clearly, at the final time $T=h / v_{0}$, we have $\Omega(T)=\Omega$ and $\Omega_{1}(T)=\Omega_{1}$.

Now, we describe the topology optimization problem which will be instrumental to optimally place the supports of the target object to be printed. The first step is to introduce the design variable which describes the material distribution. As it is common in topology optimization, it is introduced as a non-dimensional density distribution $\tilde{\rho}$. More specifically, in our framework 
we define the set of admissible densities as follows

$$
\begin{aligned}
& \mathcal{U}_{a d}=\left\{\tilde{\rho}(\mathbf{x}) \in L^{2}(\Omega): \tilde{\rho} \geq \tilde{\rho}_{\text {min }} \quad \text { a.e. in } \Omega,\right. \\
& \tilde{\rho} \leq 1 \quad \text { a.e. in } \Omega, \\
& \tilde{\rho}=\tilde{\rho}_{\text {min }} \quad \text { a.e. in } \Omega_{0} \text {, } \\
& \tilde{\rho}=1 \quad \text { a.e. in } \Omega_{1} \text {, } \\
& \left.\int_{\Omega} \tilde{\rho} \mathrm{d} \mathbf{x} \leq C\right\} .
\end{aligned}
$$

The density $\tilde{\rho}$ is bounded between $\tilde{\rho}_{\min }$ and 1 . The region where $\tilde{\rho}=1$ identifies the subdomain occupied by the material (either the object to be printed or the supports), while $\tilde{\rho}=\tilde{\rho}_{\text {min }}$ identifies the empty region. Clearly, in the region $\Omega_{1}$ occupied by the target object $\mathcal{O}$ the density is a priori fixed at one. Moreover $\tilde{\rho}$ is subject to the volume constraint $\int_{\Omega} \tilde{\rho} \mathrm{d} \mathbf{x} \leq C$, where $C$ represents the total amount of material available for the printing process.

Let $\Gamma_{D} \subset \partial \Omega$ be the portion of the boundary where the object is anchored (usually the bottom of space the domain). We require that, at each time instant $t$, the state variable $\mathbf{u}(t)$ is the solution of the following linear elasticity problem on $\Omega(t)$ written in weak for

$$
a_{\tilde{\rho}}(\mathbf{u}, \mathbf{v} ; t)=l(\mathbf{v} ; t) \quad \forall \mathbf{v} \in H_{0, \Gamma_{D}}^{1}(\Omega) \quad \text { a.e. } t \in[0, T],
$$

where we have introduced the bilinear form

$$
a_{\tilde{\rho}}(\mathbf{u}, \mathbf{v} ; t)=\int_{\Omega(t)} \mathbf{E}(\tilde{\rho}(\mathbf{x})) \nabla_{s} \mathbf{u}: \nabla_{s} \mathbf{v} \mathrm{d} \mathbf{x}
$$

and the linear functional

$$
l(\mathbf{v} ; t)=\int_{\Omega_{1}(t)} \rho_{0} \mathbf{g} \cdot \mathbf{v} \mathrm{d} \mathbf{x},
$$

being $\mathbf{E}$ the elasticity tensor, $\nabla_{s}=\frac{\nabla+\nabla^{T}}{2}$ the symmetric gradient, $\rho_{0}$ the mass density of the material used by the printer and $\mathrm{g}$ the gravity acceleration.

The particular choice of the linear functional $l$ amounts to assuming that the printed object is subject to an external load represented by the weight of the target object only (i.e. we are assuming negligible the contributions of the supports).

In the original SIMP method [2], the intermediate density values are penalized through the interpolation scheme $\mathbf{E}(\tilde{\rho}(\mathbf{x}))=\tilde{\rho}(\mathbf{x})^{p} \mathbf{E}_{0}$ (where $\mathbf{E}_{0}$ is the elasticity tensor of the considered material). The lower bound $\tilde{\rho}_{\text {min }}$ is required to avoid singularities in the elasticity problem. A modified version of the power law approach was proposed in [8]:

$$
\begin{gathered}
\mathbf{E}(\tilde{\rho}(\mathbf{x}))=\tilde{E}_{\tilde{\rho}}(\mathbf{x}) \mathbf{E}_{0} \\
\tilde{E}_{\tilde{\rho}}(\mathbf{x})=\tilde{E}_{m i n}+\tilde{\rho}(\mathbf{x})^{p}\left(1-\tilde{E}_{m i n}\right)
\end{gathered}
$$

and $0 \leq \tilde{\rho} \leq 1$. In this work, we will consider a hybrid formulation, by using (6) with $0<$ $\tilde{\rho}_{\text {min }} \leq \tilde{\rho} \leq 1$, so that we can recover the original formulation by setting $\tilde{E}_{\min }=0$, and the modified version by setting $\tilde{\rho}_{\text {min }}=0$. 


\subsection{The continuous optimization problem}

Now, we are ready to introduce our optimization problem. In particular, we ask that the unknown optimal density distribution of the supports minimizes the mean compliance of the printed object over the time interval $I$. Thus, we consider the following objective functional:

$$
\mathcal{J}(\tilde{\rho})=\frac{1}{T} \int_{0}^{T} l(\mathbf{u}(\mathbf{t}) ; t) \mathrm{d} t,
$$

where, for given density distribution $\tilde{\rho}$, the function $\mathbf{u}(\mathbf{t})$ solves the state equation (2) at each time $t$. Thus, the minimum mean compliance problem reads:

$$
\begin{array}{rl}
\min _{\tilde{\rho} \in \mathcal{U}_{a d}} & \mathcal{J}(\tilde{\rho}) \\
\text { s.t. } & \frac{1}{T} \int_{0}^{T} \int_{\Omega(t)} \tilde{E}_{\tilde{\rho}}(\mathbf{x}) \mathbf{E}_{0} \nabla_{s} \mathbf{u}(t): \nabla_{s} \mathbf{v}(t) \mathrm{d} \mathbf{x} \mathrm{d} t=\frac{1}{T} \int_{0}^{T} l(\mathbf{v} ; t) \mathrm{d} t \quad \forall \mathbf{v} \in \mathcal{V}
\end{array}
$$

It is possible to analyze the continuous time-dependent optimization problem (8), recasting the pointwise-in-time formulation (2) in an equivalent integral-in-time formulation. In this way the evolutionary problem can be addressed by employing well known solution methods for the classical SIMP problem, such as OC, MMA and CONLIN, see [1]. The analysis will be presented in [9].

\section{DISCRETIZATION OF THE PROBLEM}

In this section, we introduce the discretization of the problem (2) and we derive the discrete counterpart of the optimization problem (8) that will be solved using the OC (Optimality Conditions) method.

\subsection{Finite-element space discretization}

At the generic time instant $t$, we consider a computational grid $\mathcal{T}_{h}(t)$ partitioning the domain $\Omega(t)$ and we denote with $X_{h}(t)$ the continuous linear finite element space defined on $\mathcal{T}_{h}(t)$. We introduce the finite element space of functions compatible with the boundary conditions:

$$
V_{h}(t)=\left\{\mathbf{u}_{h} \in X_{h}(t) \quad \text { s.t. } \quad \mathbf{u}_{h}=0 \quad \text { on } \quad \Gamma_{D}\right\} .
$$

Let $\left\{\boldsymbol{\varphi}_{j}\right\}_{j=1}^{N_{h}(t)}$ be a basis of $V_{h}(t)$, where $N_{h}(t)$ is the dimension of the space. The space discretization of state equation (2) at time $t$ reads:

$$
\text { find } \begin{aligned}
& \mathbf{u}_{h}(t) \in V_{h}(t) \quad \text { s.t. } \\
& a_{\tilde{\rho}}\left(\mathbf{u}_{h}, \mathbf{v}_{h} ; t\right)=l\left(\mathbf{v}_{h} ; t\right) \quad \forall \mathbf{v}_{h} \in V_{h}(t) .
\end{aligned}
$$

By writing $\mathbf{u}_{h}(t)$ as linear combination of elements of the basis

$$
\mathbf{u}_{h}(x, t)=\sum_{j=1}^{N_{h}(t)} u_{j}(t) \boldsymbol{\varphi}_{j}
$$

we get the Galerkin approximation associated to time $t$ :

$$
\mathbf{A}_{t} \mathbf{U}_{t}=\mathbf{F}_{t}
$$

where

$$
\left[\mathbf{A}_{t}\right]_{i j}=a_{\tilde{\rho}}\left(\boldsymbol{\varphi}_{j}, \boldsymbol{\varphi}_{i} ; t\right), \quad\left[\mathbf{U}_{t}\right]_{j}=u_{j}(t), \quad\left[\mathbf{F}_{t}\right]_{i}=l\left(\boldsymbol{\varphi}_{i} ; t\right) .
$$




\subsection{Discretization in time}

We consider a uniform subdivision of the time domain $I$ and we solve the linear system (12) at the $N$ time instants $t_{n}=n T / N, n=1, \ldots, N$. The collection of vectors $\left\{\mathbf{U}_{t_{0}}, \ldots \mathbf{U}_{t_{N}}\right\}$ is the full discretization of the state variable $\mathbf{u}$ over the time interval $I$. For the sake of simplicity, we consider a structured grid with a vertical discretization such that the grid $\mathcal{T}_{h}$ on the full space domain $\Omega$ can be split into the $N$ horizontal layers $E \times\left[v_{0} t_{n-1}, v_{0} t_{n}\right]$ for $n=1, \ldots N$. Under this hypothesis, given $\mathcal{T}_{h}$ and a basis $\left\{\boldsymbol{\varphi}_{j}\right\}_{j \in V}$ of the finite element space $V_{h}$ defined on $\mathcal{T}_{h}$, we can recover $V_{h}(t)$ as the space generated by the subset of $\left\{\boldsymbol{\varphi}_{j}\right\}_{j \in V}$ of functions whose support has non-empty intersection with $\Omega\left(t_{n}\right)$.

When multiple layers $(N>1)$ are used in the computation of the optimal time-averaged compliance, the resulting approach will be referred to as multi-layer approach. Otherwise, for $N=1$ we recover the standard minimal compliance optimization that we will refer to as single-layer approach.

\subsection{Full discretization}

Let $K_{e}$ be a generic element of the mesh $\mathcal{T}_{h}$, with index $e \in B$. We denote with $B^{0}$ and $B^{1}$ the sets of indexes of elements belonging to $\Omega_{0}$ and $\Omega_{1}$, respectively. The subset of mesh elements contained in the domain $\Omega\left(t_{n}\right)$ is denoted as $B_{n} \subset B$. Moreover, let $n_{e}$ indicate the value of the smallest time step such that the element $K_{e}$ belongs to $\Omega\left(t_{n}\right)$ and let $V_{e}$ be the set of indexes of degrees of freedom associated with the element $e$. Thus the following relations hold:

$$
\begin{aligned}
& \bigcup_{e \in B} K_{e}=\Omega, \\
& \bigcup_{e \in B_{n}} K_{e}=\Omega\left(t_{n}\right), \\
& e \in B_{n} \Longleftrightarrow n \geq n_{e} .
\end{aligned}
$$

We remark that the finite element approximation $\rho_{h}$ of the density is piecewise constant over the triangulation $\mathcal{T}_{h}$, while the state variable $\mathbf{u}$ is discretized by continuous in space (with basis $\left.\left\{\boldsymbol{\varphi}_{j}\right\}_{j \in V}\right)$ piecewise constant in time finite elements, i.e.

$$
\begin{aligned}
\rho_{h}(\mathbf{x}) & =\sum_{e \in B} \tilde{\rho}_{e} \mathbb{1}_{K_{e}}(\mathbf{x}), \\
\mathbf{u}_{h}(\mathbf{x}, t) & =\sum_{n=1}^{N} \sum_{j \in V} u_{j}^{n} \boldsymbol{\varphi}_{j}(\mathbf{x}) \mathbb{1}_{\left(t_{n-1}, t_{n}\right]}(t) .
\end{aligned}
$$

We define

$$
\begin{aligned}
K_{i j}^{e} & =\int_{K_{e}} \mathbf{E}_{0} \nabla_{s} \boldsymbol{\varphi}_{i}: \nabla_{s} \boldsymbol{\varphi}_{j} \mathrm{~d} \mathbf{x}, \\
f_{i}^{e} & =\int_{K_{e}} \rho_{0} \mathbf{g} \cdot \boldsymbol{\varphi}_{i} \mathrm{~d} \mathbf{x},
\end{aligned}
$$

and the following SIMP interpolation holds:

$$
\tilde{E}\left(\tilde{\rho}_{e}\right)=\tilde{E}_{m i n}+\tilde{\rho}_{e}^{p}\left(1-\tilde{E}_{m i n}\right) .
$$


Finally, the constraint $1 \mathrm{e})$ reads as follows:

$$
\sum_{e \in B}\left|K_{e}\right| \tilde{\rho}_{e} \leq C
$$

In view of the above discussion, the discrete counterpart of the minimization problem (8) reads as follows:

$$
\begin{aligned}
\min _{\tilde{\rho}_{e}} & \frac{1}{N} \sum_{n=1}^{N} \sum_{e \in B_{n}} \mathbb{1}_{B^{1}}(e) \sum_{i \in V_{e}} f_{i}^{e} u_{i}^{n} \\
\text { s.t. } & \frac{1}{N} \sum_{n=1}^{N} \sum_{e \in B_{n}} \tilde{E}\left(\tilde{\rho}_{e}\right) \sum_{i, j \in V_{e}} K_{i j}^{e} u_{i}^{n} v_{j}^{n} \\
& =\frac{1}{N} \sum_{n=1}^{N} \sum_{e \in B_{n}} \mathbb{1}_{B^{1}}(e) \sum_{i \in V_{e}} f_{i}^{e} v_{i}^{n} \quad \forall\left\{v_{i}^{n}\right\}_{n=1, \ldots, N}^{i \in V} \subset \mathbb{R} \\
& \sum_{e \in B}\left|K_{e}\right| \tilde{\rho}_{e} \leq C \\
& \tilde{\rho}_{\text {min }} \leq \tilde{\rho}_{e} \leq 1 \quad \forall e \in B \\
& \tilde{\rho}_{e}=\tilde{\rho}_{\text {min }} \quad \forall e \in B^{0} \\
& \tilde{\rho}_{e}=1 \quad \forall e \in B^{1} .
\end{aligned}
$$

The Lagrangian function for the discretized problem is defined as follows:

$$
\begin{aligned}
\mathcal{L} & =\frac{1}{N} \sum_{n=1}^{N} \sum_{e \in B_{n}} \mathbb{1}_{B^{1}}(e) \sum_{i \in V_{e}} f_{i}^{e} u_{i}^{n}-\frac{1}{N} \sum_{n=1}^{N} \sum_{e \in B_{n}}\left(\tilde{E}\left(\tilde{\rho}_{e}\right) \sum_{i, j \in V_{e}} K_{i j}^{e} u_{i}^{n} u_{j}^{n}-\mathbb{1}_{B^{1}}(e) \sum_{i \in V_{e}} f_{i}^{e} u_{i}^{n}\right) \\
& +\Lambda\left(\sum_{e \in B}\left|K_{e}\right| \tilde{\rho}_{e}-C\right)+\sum_{e \in B} \lambda_{e}^{+}\left(\tilde{\rho}_{e}-1\right)+\sum_{e \in B} \lambda_{e}^{-}\left(\tilde{\rho}_{\text {min }}-\tilde{\rho}_{e}\right) .
\end{aligned}
$$

where $\Lambda$ is the Lagrangian multiplier for the constraint $(1 \mathrm{e}), \lambda_{e}^{+}$for the constraint $(1 \mathrm{~b})$ and $\lambda_{e}^{-}$ for (1a). The constraints (1c) are (1d) are not plugged in the Lagrangian, but will be considered later by projecting the solution on the space $\mathcal{U}_{a d}$ in (24).

If we derive the Lagrangian with respect to the state variable we get the discrete version of adjoint equation, while deriving with respect to the design variables $\tilde{\rho}_{e}$ we get:

$$
\frac{\partial \mathcal{L}}{\partial \tilde{\rho}_{e}}(\xi)=\sum_{e \in B}\left[-\frac{1}{N} \sum_{n=n_{e}}^{N}\left(p \tilde{\rho}_{e}^{p-1}\left(1-\tilde{E}_{m i n}\right) \sum_{i, j \in V_{e}} K_{i j}^{e} u_{i}^{n} u_{j}^{n}\right)+\Lambda\left|K_{e}\right|+\lambda_{e}^{+}-\lambda_{e}^{-}\right] \xi
$$

By defining

$$
\Psi_{e}=\frac{1}{N\left|K_{e}\right|} \sum_{n=n_{e}}^{N}\left(p \tilde{\rho}_{e}^{p-1}\left(1-\tilde{E}_{\text {min }}\right) \sum_{i, j \in V_{e}} K_{i j}^{e} u_{i}^{n} u_{j}^{n}\right)
$$

we get the optimality conditions for the discretized problem which reads as follows

$$
\begin{cases}\Psi_{e}=\Lambda & \text { if } \tilde{\rho}_{\min }<\tilde{\rho}_{e}<1 \\ \Psi_{e} \leq \Lambda & \text { if } \tilde{\rho}_{e}=\tilde{\rho}_{\min } \\ \Psi_{e} \geq \Lambda & \text { if } \tilde{\rho}_{e}=1\end{cases}
$$




\subsection{A fixed point algorithm}

Based on (23), it is possible to derive an Optimality Condition (OC) method that computes a sequence $\left\{\tilde{\rho}_{e}^{K}\right\}$ approximating $\tilde{\rho}_{e}$ by resorting to the following fixed-point algorithm: for $K \geq 1$

$$
\tilde{\rho}_{e}^{K+1}= \begin{cases}\tilde{\rho}_{\text {min }} & \text { if } e \in B^{0} \\ 1 & \text { if } e \in B^{1} \\ \max \left\{(1-\zeta) \tilde{\rho}_{e}^{K}, \tilde{\rho}_{\text {min }}\right\} & \text { if } \tilde{\rho}_{e}^{K}\left(Q_{e}^{K}\right)^{\eta} \leq \max \left\{(1-\zeta) \tilde{\rho}_{e}^{K}, \tilde{\rho}_{\text {min }}\right\}, e \notin\left(B^{1} \cup B^{0}\right) \\ \min \left\{(1+\zeta) \tilde{\rho}_{e}^{K}, 1\right\} & \text { if } \tilde{\rho}_{e}^{K}\left(Q_{e}^{K}\right)^{\eta} \geq \min \left\{(1+\zeta) \tilde{\rho}_{e}^{K}, 1\right\}, e \notin\left(B^{1} \cup B^{0}\right) \\ \tilde{\rho}_{e}^{K}\left(Q_{e}^{K}\right) i^{\eta} & \text { elsewhere }\end{cases}
$$

where $Q_{e}^{K}=\Psi_{e}^{K} / \Lambda^{K}$.

Since the space integral of updated density is a continuous non decreasing function of the multiplier $\Lambda$, its updated value $\Lambda^{K}$ can be computed by employing a bisection algorithm. The variable $\zeta$ is a move limit, and $\eta$ is a tuning parameter. Both values can be adjusted to improve efficiency of the algorithm. Typical values are respectively 0.1 and 0.5 . In the actual implementation some filtering procedure must be taken into account to get a well-posed problem, see e.g. [7].

\subsection{Description of the algorithm}

In this section, we briefly summarize the different steps required to apply the proposed methodology, from the pre-processing needed to setup the simulation to the actual optimization loop.

\section{Pre-processing}

- Choose a cylindric reference space domain $\Omega$ and identify sub-domains $\Omega_{0}$ and $\Omega_{1}$.

- Subdivide time interval $[0, T]$ in $N$ time steps.

- Build a spatial mesh $\mathcal{T}_{h}$ on $\Omega$, fine enough to describe the geometrical details of the sub-domains $\Omega_{0}$ and $\Omega_{1}$ and conforming with the horizontal layers at $y=$ $v_{0} t_{1}, \ldots, v_{0} t_{N}$.

- Build the finite element space $V_{h}$ with its basis functions.

- Choose an initial design for the variables $\tilde{\rho}_{e}$ (for instance a uniform distribution).

\section{Optimization}

- For each time step $t_{n}$, compute the displacement field with the current value of the design variable (see (12)).

- Compute for each mesh element the value of $\Psi_{e}$ according to (22).

- Compute the current value of $\Lambda_{K}$ by bisection.

- Update the design variables $\tilde{\rho}_{e}$ as in (24).

- Repeal until a stopping criterion is satisfied. 


\section{NUMERICAL RESULTS}

In this section we present some numerical test cases to assess the properties of the proposed topology optimization scheme for the design of support structures. In particular, we will highlight the differences between the solution obtained with the proposed multi-layer approach minimizing the mean compliance during the printing process and the one obtained from a standard single-layer minimal compliance problem for the whole object.

\subsection{Test case 1}

In the first test case we consider a self-supporting structure defined by an inclined tapered beam with the thinner extreme joint to the ground and the thicker extreme supported by a vertical pillar (in black in Fig 2). The domain is $\Omega=(0,1) \times(0,0.5)$ and the prescribed active region $\Omega \backslash \Omega_{0} \cup \Omega_{1}$ (where the supports can be placed) is the area below the structure (in grey in Fig. 2. Both the object and the supports are made of thermoplastic polyurethane with $\rho_{0}=1.1 \cdot 10^{3}$ $\mathrm{Kg} / \mathrm{m}^{3}$ and $E=3 \cdot 10^{7} \mathrm{~Pa}$. The optimization was performed on three different mesh resolutions $(50 \times 25,100 \times 50,200 \times 100)$ using a sensitivity filter with a fixed filter size of 0.2 (independent of the mesh size). The results obtained using a standard single-layer approach for the final object shape are reported in Fig. 3 (left), while the results of the proposed multi-layer using 25 time intervals are reported in Fig. 3 (right).

First, we note that in both approaches the main features of the solution are captured even with a coarse grid and the solution is robust as the space resolution is increased. However, position and size of the supports generated by the two approaches are different. In particular, for the multi-layer approach the supports are shifted on the right and thickened in order to reduce the compliance in the first phases of the printing process when the tapered beam is not yet self-supporting.

In order to better understand the reasons behind the difference between the optimal solutions obtained with the two approaches, we have evaluated, for the two configurations, the history of the compliance during the printing process by solving a sequence of 50 linear elasticity problems, each one at a different time instant belonging to the time interval $I$. The results presented in Fig. 4 clearly show that the shape optimized using the multi-layer approach guarantees a significant reduction of the compliance over a large portion of the time interval considered. Obviously, the value of the compliance at the final time is lower for the single-layer approach since in this case the compliance at $t=T$ is exactly the functional that is minimized.

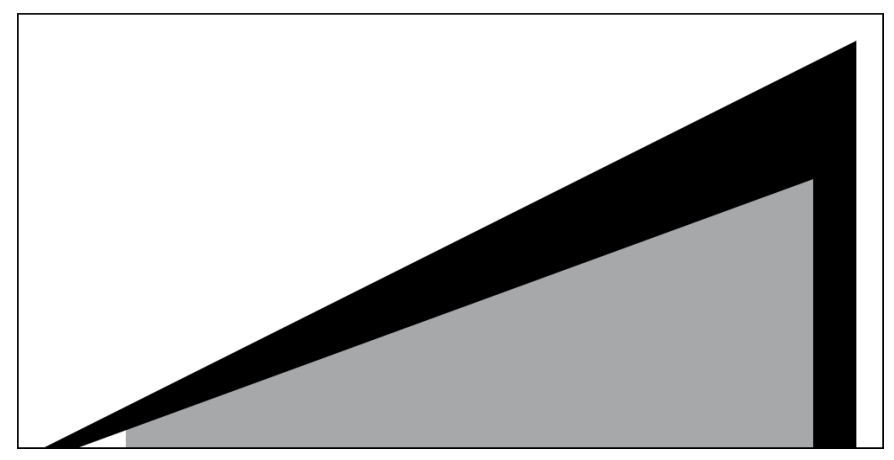

Figure 2: Computational domain for test case 1 

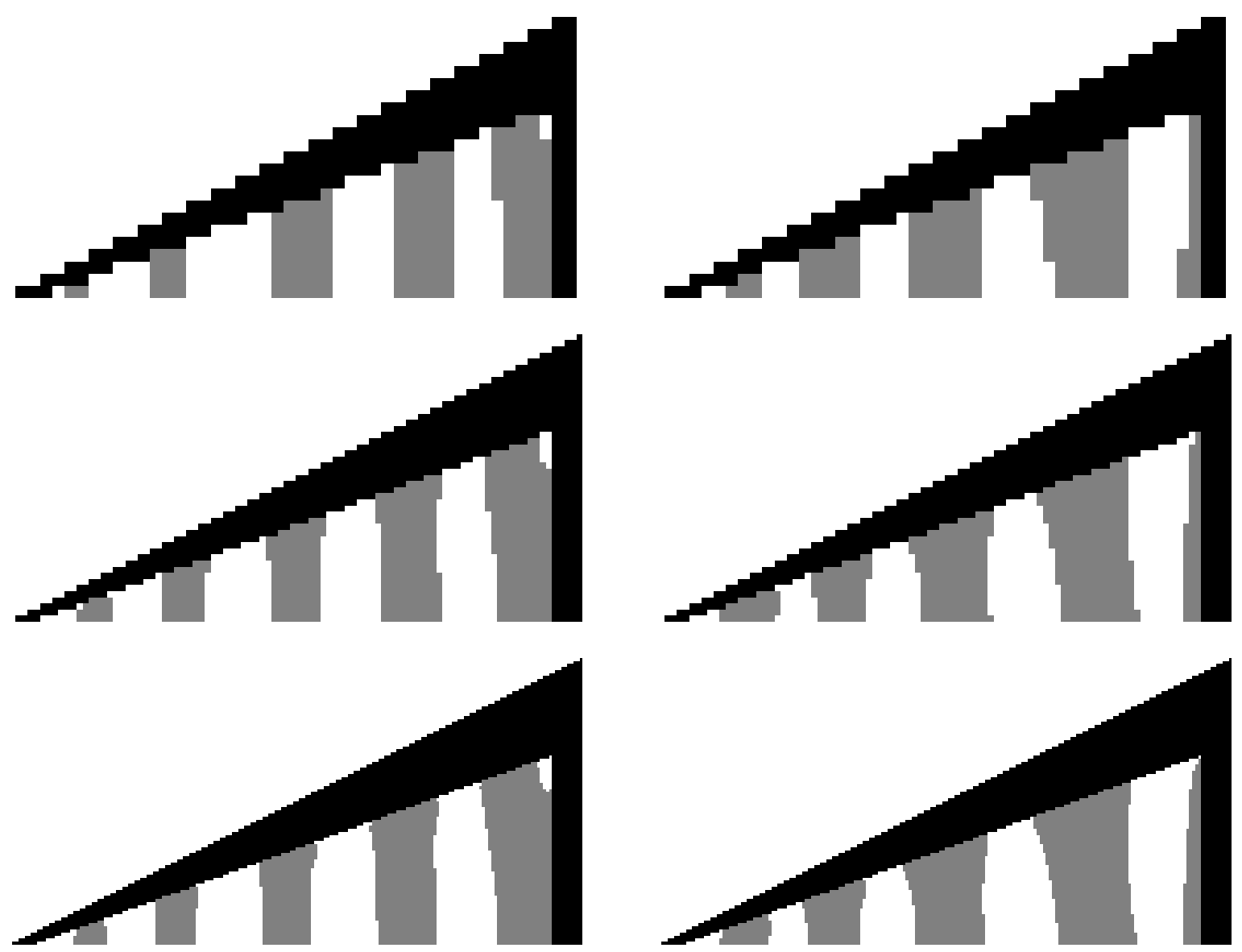

Figure 3: Results of test case 1: single-layer minimization (left), multi-layer minimization (right). Increasing mesh resolution from top to bottom.

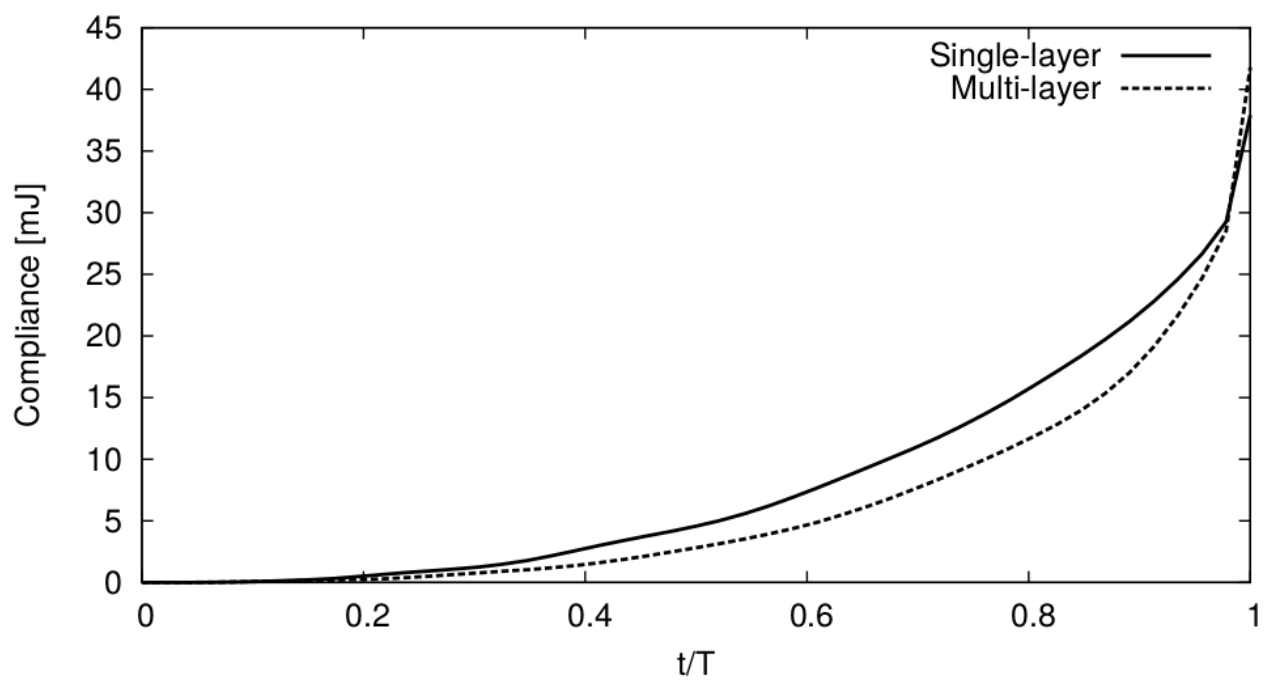

Figure 4: Time-evolution of the compliance for the two configurations obtained by the single-layer (solid line) and multi-layer (dashed-line) approaches in test case 1. 


\subsection{Test case 2}

In the second test case, with the aim of precisely identifying the conditions under which our novel approach is more appropriate than the standard one, we continue the analysis of the differences between the single-layer compliance minimization and the multi-layer mean compliance minimization (on 25 time instants). We consider the two structures (squared and rounded) displayed in black in Fig. 5 which are fixed on the ground. The domain is $\Omega=$ $(0,1) \times(0,0.25)$ and the prescribed active region where the support can be added is identified by the grey region below the structure. The material properties and filtering procedure are the same as for test case 1 .
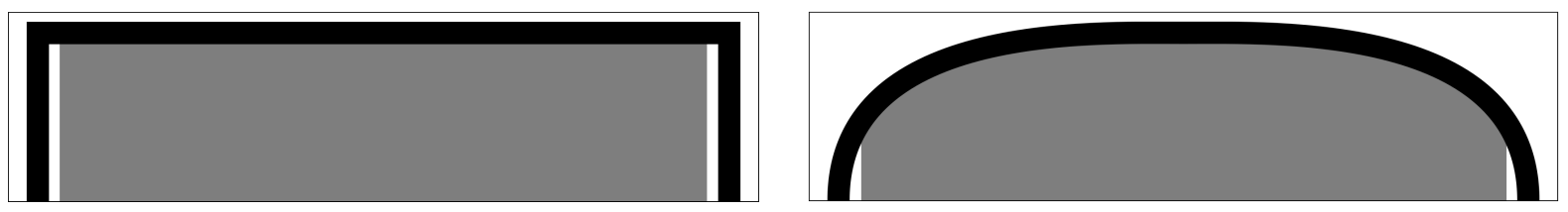

Figure 5: Computational domains for test case 2: squared structure (left) and rounded structure (right)

Let us first consider the squared structure and compare the results of the support optimization obtained by using the single-layer or the multi-layer approach (see Fig. 6). Differently from the previous test case, here the optimal supports obtained by the two approaches are almost identical. This is not surprising, due to the particular geometry of the squared structure. Indeed, only the last few layers give a significant contribution to the mean compliance, so that the functionals to be minimized by the two approaches are quite similar.

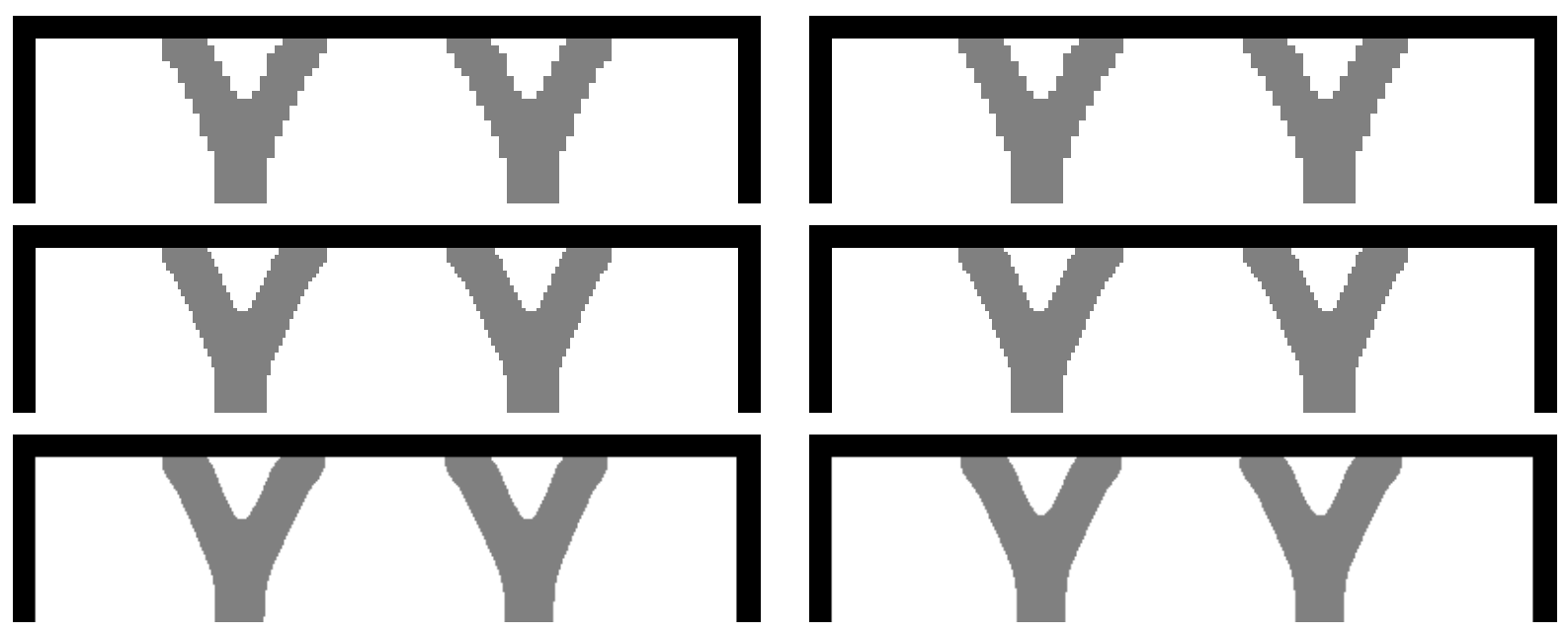

Figure 6: Results of test case 2 (squared structure): single-layer minimization (left), multi-layer minimization (right). Increasing mesh resolution from top to bottom.

Remarkably, when the rounded structure is considered, the two approaches lead to optimal solutions which are very different as displayed in Fig. 7. Here a double tree-like structure for the single-layer approach and a pillar structure for the multi-layer approach are obtained. In the latter case, at the early stages of the printing process, two overhanging structures need to be supported and this fact drives the optimization towards a structure composed by six supporting structures distributed non-uniformly along the domain width. 


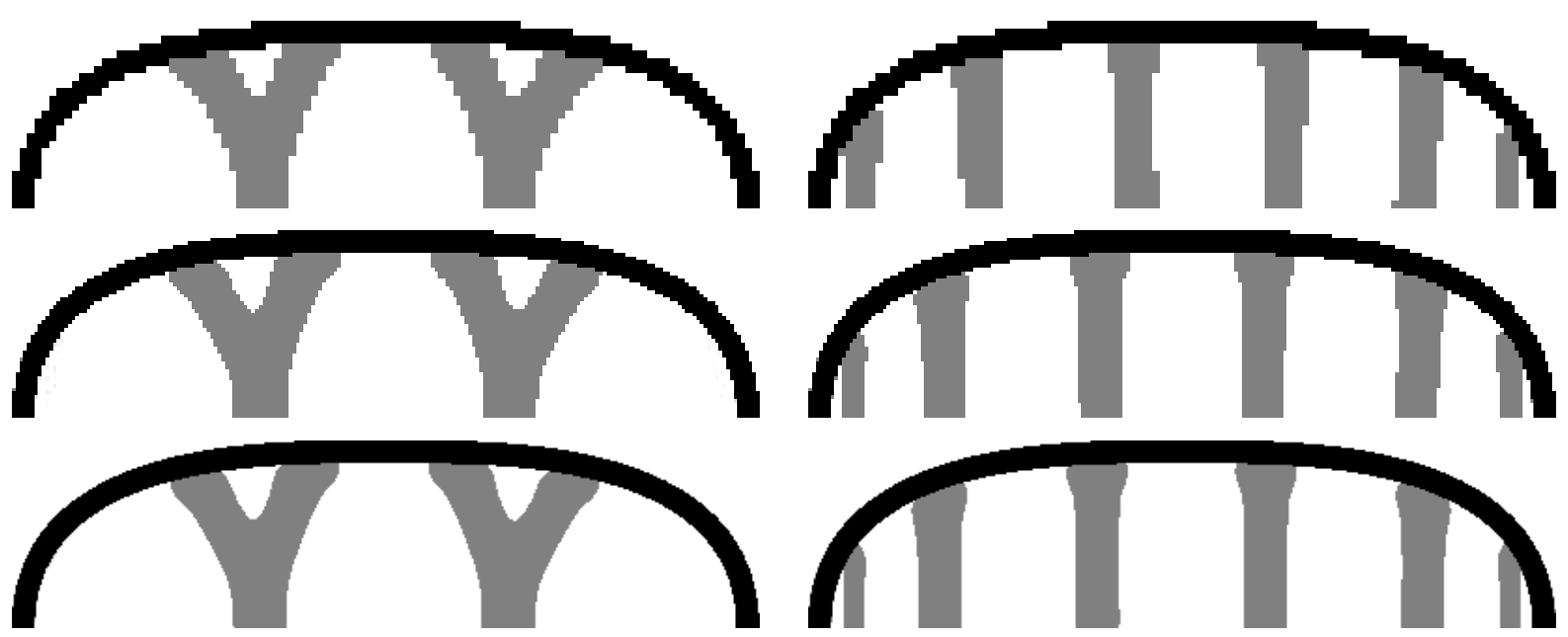

Figure 7: Results of test case 2 (rounded structure): single-layer minimization (left), multi-layer minimization (right). Increasing mesh resolution from top to bottom.

\section{CONCLUSIONS}

We have presented a new topology optimization algorithm for time-dependent linear elasticity problems. The algorithm has been devised for facing the time-dependent nature of additive manufacturing processes, with the aim of computing the optimal material distribution which minimizes the mean compliance over a time interval.

The algorithm has been derived applying the Optimality Condition framework to the timedependent elasticity problem leading to the introduction of a fixed-point iterative scheme.

Numerical tests have been performed with the aim of investigating the properties of the proposed approach. Moreover, a detailed comparison with the standard minimal compliance optimization has been carried out. The results show that our method is reliable in handling situations typical in additive manufacturing where overhangs appear in the early stages of the printing process aiming at the production of self-sustained structures.

\section{ACKNOWLEDGEMENTS}

The authors gratefully acknowledge Moxoff s.r.l. for pointing out this interesting topic and for the partial support to this research project.

\section{REFERENCES}

[1] M. P. Bendsøe, O. Sigmund, Topology optimization theory, methods and applications, New York, Springer, 2003.

[2] M. P. Bendsøe, Optimal shape design as a material distribution problem, Structural Optimization, 1 (1989), 193-202.

[3] K. Svanberg, Method of moving asymptotes - a new method for structural optimization, International Journal for Numerical Methods in Engineering, 24 (1897), 193-202.

[4] T. Zegard, G. H. Paulino, Bridging topology optimization and additive manufacturing, Structural and Multidisciplinary Optimization, 53(1) (2016), 175-192. 
[5] R. Rezaie, M. Badrossamay, A. Ghaei, H. Moosavi, Topology optimization for fused deposition modeling process, Paper presented at the Procedia CIRP, 6 (2013), 521-526.

[6] A. T. Gaynor, N. A. Meisel, C. B. Williams, J. K. Guest, Multiple-material topology optimization of compliant mechanisms created via PolyJet three-dimensional printing, Journal of Manufacturing Science and Engineering, Transactions of the ASME, 136(6) (2014), 110.

[7] O. Sigmund O, J. Petersson, Numerical instabilities in topology optimization: a survey on procedures dealing with checkerboards, mesh-dependencies and local minima, Structural Optimization, 16 (1998), 68-75.

[8] O. Sigmund, Morphology-based black and white filters for topology optimization, Structural and Multidisciplinary Optimization 33 (4-5) (2007), 401-424.

[9] M. Bruggi, N. Parolini, F. Regazzoni, M. Verani, Time-dependent topology optimization: an application to additive manufacturing processes. In preparation. 